\author{
YAÉ UlRich GABA
}

\title{
SOME ADVANCES IN THE THEORY OF QUASI-PSEUDOMETRIC TYPE SPACES
}

\begin{abstract}
In this paper, we extend most of the results proved in [4]. In particular, we give some topological properties of the quasi-pseudometric type spaces. Moreover, some fixed point and common fixed point theorems are obtained in the setting of quasi-pseudometric spaces, introduced some months ago by Kazeem et al in [4].
\end{abstract}

KEY WORDS: quasi-pseudometric type spaces, fixed point, left $K$-completeness.

AMS Mathematics Subject Classification: $47 \mathrm{H} 09$.

Symmetric spaces were introduced in 1931 by Wilson [6], as metric-like spaces lacking the triangle inequality. Several fixed point results in such spaces were obtained. In the same dynamics, cone metric spaces were introduced by Huang [3] and many fixed point results concerning mappings in these spaces have also been established. In [5], M. A. Khamsi connected this concept with a generalised form of metric that he named metric type. Namely, he observed that if $d(x, y)$ is a cone metric, then $D(x, y)=\|d(x, y)\|$ is symmetric with some special properties, particularly in the case when the underlying cone is normal. Recently in [4], Kazeem et al. discussed the newly introduced notion of quasi-pseudometric type spaces as a logical equivalent to metric type spaces when the initial distance-like function is not symmetric. Some fixed point results of mappings on such spaces were discussed as well in [4]. It is the aim of this article to continue the study of quasi-pseudometric spaces by proving several other fixed point and common fixed point results, hence extending the fixed point results of [4] to a class of mappings satisfying more general contractive conditions.

In this section, we recall briefly some elementary definitions from the asymmetric topology which are necessary for a good understanding of the work below. For recent results and detailed explanations for the concepts in the theory of asymmetric spaces, the reader is referred to $[2,4,7,8]$. 
Definition 1. Let $E$ be a real Banach space with norm $\|$.$\| and P$ be a subset of $E$. Then $P$ is called a cone if and only if

(a) $P$ is closed, nonempty and $P \neq\{\theta\}$, where $\theta$ is the zero vector in $E$;

(b) for any $a, b \geq 0$, and $x, y \in P$, we have $a x+b y \in P$;

(c) for $x \in P$, if $-x \in P$, then $x=\theta$.

Given a cone $P$ in a Banach space $E$, we define on $E$ a partial order $\preceq$ with respect to $P$ by

$$
x \preceq y \Longleftrightarrow y-x \in P .
$$

We also write $x \prec y$ whenever $x \preceq y$ and $x \neq y$, while $x \ll y$ will stand for $y-x \in \operatorname{Int}(P)$ (where $\operatorname{Int}(P)$ designates the interior of $P$ ).

The cone $P$ is called normal if there is a number $C>0$, such that for all $x, y \in E$, we have

$$
\theta \preceq x \preceq y \Longrightarrow\|x\| \leq C\|y\|
$$

The least positive number satisfying this inequality is called the normal constant of $P$. Therefore, we shall then say that $P$ is a $K$-normal cone to indicate the fact that the normal constant is $K$.

Definition 2 (Compare [4]). Let $X$ be a nonempty set. Suppose the mapping $q: X \times X \rightarrow E$ satisfies

(q1) $\theta \preceq q(x, y)$ for all $x, y \in X$;

(q2) $q(x, y)=\theta=q(y, x)$ if and only if $x=y$;

(q3) $q(x, z) \preceq q(x, y)+q(y, z)$ for all $x, y, z \in X$.

Then, $q$ is called a quasi-cone metric on $X$, and $(X, q)$ is called a quasi-cone metric space.

Definition 3 (Compare [4]). A sequence in a quasi-cone metric space $(X, q)$ is called

(a) Q-Cauchy or bi-Cauchy if for every $c \in X$ with $c \gg \theta$, there exists $n_{0} \in \mathbb{N}$ such that

$$
\forall n, m \geq n_{0} \quad q\left(x_{n}, x_{m}\right) \ll c ;
$$

(b) left(right) Cauchy if for every $c \in X$ with $c \gg \theta$, there exists $n_{0} \in \mathbb{N}$ such that

$$
\forall n, m: n_{0} \leq m \leq n \quad q\left(x_{m}, x_{n}\right) \ll c\left(q\left(x_{n}, x_{m}\right) \ll c \text { resp. }\right) .
$$

Remark 1. A sequence is $Q$-Cauchy if and only if it is both left and right Cauchy.

Definition 4. (a) In a quasi-cone metric space $(X, q)$, we say that the sequence $\left(x_{n}\right)$ left converges to $x \in X$ if for every $c \in E$ with $\theta \ll c$ there exists $N$ such that for all $n>N, q\left(x_{n}, x\right) \ll c$. 
(b) Similarly, in a quasi-cone metric space $(X, q)$, we say that a sequence $\left(x_{n}\right)$ right converges to $x \in X$ if for every $c \in E$ with $\theta \ll c$ there exists $N$ such that for all $n>N, q\left(x, x_{n}\right) \ll c$.

(c) Finally, in a quasi-cone metric space $(X, q)$, we say that the sequence $\left(x_{n}\right)$ converges to $x \in X$ if for every $c \in E$ with $\theta \ll c$ there exists $N$ such that for all $n>N, q\left(x_{n}, x\right) \ll c$ and $q\left(x, x_{n}\right) \ll c$.

Definition 5. A quasi-cone metric space $(X, q)$ is called

(a) left complete (resp. right complete) if every left Cauchy (resp. right Cauchy) sequence in $X$ left (resp. right) converges.

(b) bicomplete if every $Q$-Cauchy sequence converges.

Remark 2. A quasi-cone metric space $(X, q)$ is bicomplete if and only if it is left complete and right complete.

Definition 6. Let $(X, q)$ be a quasi-cone metric space. A function $f$ : $X \rightarrow X$ is said to be Lipschitzian if there exists some $\kappa \in \mathbb{R}$ such that

$$
q(f(x), f(y)) \preceq \kappa q(x, y) \quad \forall x, y \in X .
$$

The smallest constant which satisfies the above inequality is called the Lipschitiz constant of $f$ and is denoted Lip $(f)$. In particular $f$ is said to be contractive if $\operatorname{Lip}(f) \in[0,1)$ and nonexpansive if $\operatorname{Lip}(f) \leq 1$.

Definition 7 (Compare [1]). Let $f$ and $g$ be self maps on a set $X$. If $w=f x=g x$ for some $x \in X$, then $x$ is called a coincidence point of $f$ and $g$, and $w$ is called the point of coincidence of $f$ and $g$.

Definition 8. Let $f$ and $g$ be self maps on a nonempty set $X$. We say that $f$ and $g$ are weakly compatible if they commute at their coincidence point, that is there exists $x_{0} \in X$ such that $f x_{0}=g x_{0}$ then $g f x_{0}=f g x_{0}$.

We also give the following proposition that we take from [1] by omitting the proof.

Proposition 1 (Compare [1]). Let $f$ and $g$ be weakly compatible self maps on a set $X$. If $f$ and $g$ have a unique point of coincidence $w=f x=g x$, then $w$ is the unique common fixed point of $f$ and $g$.

we also have the following important characterization

Lemma 1. Let $(X, q)$ be a quasi-cone metric space, $P$ be a $K$-normal cone and $\left(x_{n}\right)$ be a sequence in $X$. Then $\left(x_{n}\right)$ is a bi-Cauchy sequence if and only if $q\left(x_{n}, x_{m}\right) \longrightarrow \theta$ as $n, m \longrightarrow \infty$.

We now connect the notion of quasi-cone metric to the one of quasi-pseudometric type space via the following theorem. 
Theorem 1 (Compare [4] Theorem 28). Let $(X, q)$ be a quasi-cone metric space over the Banach space $E$ with the $K$-normal cone $P$. The mapping $Q: X \times X \rightarrow[0, \infty)$ defined by $Q(x, y)=\|q(x, y)\|$ satisfies the following properties

(Q1) $Q(x, x)=0$ for any $x \in X$;

$(Q 2) Q(x, y) \leq K\left(Q\left(x, z_{1}\right)+Q\left(z_{1}, z_{2}\right)+\cdots+Q\left(z_{n}, y\right)\right)$, for any points $x, y, z_{i} \in X, i=1,2, \ldots, n$.

We are therefore led to the following definition.

Definition 9 ([4]). Let $X$ be a non empty set, and let the function $D: X \times X \rightarrow[0, \infty)$ satisfy the following properties:

(D1) $D(x, x)=0$ for any $x \in X$;

(D2) $D(x, y) \leq \alpha\left(D\left(x, z_{1}\right)+D\left(z_{1}, z_{2}\right)+\cdots+D\left(x_{n}, y\right)\right)$ for any points $x, y, z_{i} \in X, i=1,2, \ldots, n$ and some constant $\alpha>0$.

Then $(X, D, \alpha)$ is called a quasi-pseudometric type space. Moreover, if $D(x, y)=0=D(y, x) \Longrightarrow x=y$, then $D$ is said to be a $T_{0}$-quasi-pseudometric type space. The latter condition is referred to as the $T_{0}$-condition.

Remark 3. - Let $D$ be a quasi-pseudometric type on $X$, then the map $D^{-1}$ defined by $D^{-1}(x, y)=D(y, x)$ whenever $x, y \in X$ is also a quasi-pseudometric type on $X$, called the conjugate of $D$. We shall also denote $D^{-1}$ by $D^{t}$ or $\bar{D}$.

- It is easy to verify that the function $D^{s}$ defined by $D^{s}:=D \vee D^{-1}$, i.e. $D^{s}(x, y)=\max \{D(x, y), D(y, x)\}$ defines a metric type (see [5]) on $X$ whenever $D$ is a $T_{0}$-quasi-pseudometric type.

- If we substitute the property $(D 1)$ by the following property $(D 3): D(x, y)=0 \Longleftrightarrow x=y$,

we obtain a $T_{0}$-quasi-pseudometric type space directly. For instance, this could be done if the map $D$ is obtained from quasi-cone metric.

Moreover, for $\alpha=1$, we recover the classical pseudometric, hence quasipseud-metric type spaces generalize quasi-pseudometrics. It is worth mentioning that if $(X, D, \alpha)$ is a pseudometric type space, then for any $\beta \geq \alpha$, $(X, D, \beta)$ is also a pseudometric type space. We give the following example to illustrate the above comment.

Example 1. Let $X=\{a, b, c\}$ and the mapping $D: X \times X \rightarrow[0, \infty)$ defined by $D(a, b)=D(c, b)=1 / 5, D(b, c)=D(b, a)=D(c, a)=1 / 4, D(a, c)=$ $1 / 2, D(x, x)=0$ for any $x \in X$ and $D(x, y)=D(y, x)$ for any $x, y \in X$. Since

$$
\frac{1}{2}=D(a, c)>D(a, b)+D(b, c)=\frac{9}{20},
$$


then we conclude that $X$ is not a quasi-pseudometric space. Nevertheless, with $\alpha=2$, it is very easy to check that $(X, D, 2)$ is a quasi-pseudometric type space.

Definition $10([4])$. Let $(X, D, \alpha)$ be a quasi-pseudometric space. The convergence of a sequence $\left(x_{n}\right)$ to $x$ with respect to $D$, called $D$-convergence or left-convergence and denoted by $x_{n} \stackrel{D}{\longrightarrow} x$, is defined in the following way

$$
x_{n} \stackrel{D}{\longrightarrow} x \Longleftrightarrow D\left(x, x_{n}\right) \longrightarrow 0 .
$$

Similarly, the convergence of a sequence $\left(x_{n}\right)$ to $x$ with respect to $D^{-1}$, called $D^{-1}$-convergence or right-convergence and denoted by $x_{n} \stackrel{D^{-1}}{\longrightarrow} x$, is defined in the following way

$$
x_{n} \stackrel{D^{-1}}{\longrightarrow} x \Longleftrightarrow D\left(x_{n}, x\right) \longrightarrow 0 .
$$

Finally, in a quasi-pseudometric space $(X, D, \alpha)$, we shall say that a sequence $\left(x_{n}\right) D^{s}$-converges to $x$ if it is both left and right convergent to $x$, and we denote it as $x_{n} \stackrel{D^{s}}{\longrightarrow} x$ or $x_{n} \longrightarrow x$ when there is no confusion. Hence

$$
x_{n} \stackrel{D^{s}}{\longrightarrow} x \Longleftrightarrow x_{n} \stackrel{D}{\longrightarrow} x \text { and } x_{n} \stackrel{D^{-1}}{\longrightarrow} x \text {. }
$$

Definition $11([4])$. A sequence $\left(x_{n}\right)$ in a quasi-pseudometric type space $(X, D, \alpha)$ is called

(a) left $K$-Cauchy with respect to $D$ if for every $\epsilon>0$, there exists $n_{0} \in \mathbb{N}$ such that

$$
\forall n, k: n_{0} \leq k \leq n \quad D\left(x_{k}, x_{n}\right)<\epsilon ;
$$

(b) right $K$-Cauchy with respect to $D$ if for every $\epsilon>0$, there exists $n_{0} \in \mathbb{N}$ such that

$$
\forall n, k: n_{0} \leq k \leq n \quad D\left(x_{n}, x_{k}\right)<\epsilon ;
$$

(c) $D^{s}$-Cauchy if for every $\epsilon>0$, there exists $n_{0} \in \mathbb{N}$ such that

$$
\forall n, k \geq n_{0} \quad D\left(x_{n}, x_{k}\right)<\epsilon .
$$

Remark 4. - A sequence is left $K$-Cauchy with respect to $d$ if and only if it is right $K$-Cauchy with respect to $D^{-1}$.

- A sequence is $d^{s}$-Cauchy if and only if it is both left and right $K$-Cauchy.

Definition 12 ([4]). A quasi-pseudometric space $(X, D, \alpha)$ is called left-complete provided that any left $K$-Cauchy sequence is D-convergent. 
Definition 13 ([4]). A quasi-pseudometric space $(X, D, \alpha)$ is called right-complete provided that any right $K$-Cauchy sequence is D-convergent.

Definition 14 ([4]). A $T_{0}$-quasi-pseudometric space $(X, D, \alpha)$ is called bicomplete provided that the metric $D^{s}$ on $X$ is complete.

\section{First results}

In [4], Kazeem et al. proved the following:

Theorem 2. Let $(X, q)$ be a bicomplete quasi-cone metric space, $P$ a $K$-normal cone. Suppose that a mapping $T: X \rightarrow X$ satisfies the contractive condition

$$
q(T x, T y) \preceq k q(x, y) \text { for all } x, y \in X,
$$

where $k \in[0,1)$. Then $T$ has a unique fixed point. Moreover for any $x \in X$, the orbit $\left\{T^{n} x, n \geq 0\right\}$ converges to the fixed point.

We start by an application of the above the theorem

Theorem 3. Let $(X, q)$ be a bicomplete quasi-cone metric space, $P$ a $K$-normal cone. Let $T: X \rightarrow X$ be a map such that for every $n \in \mathbb{N}$, there is $\lambda_{n} \in(0,1)$ such that

$$
q\left(T^{n} x, T^{n} y\right) \preceq \lambda_{n} q(x, y) \text { for all } x, y \in X .
$$

and let $\lim _{n \rightarrow 0} \lambda_{n}=0$. Then $T$ has a unique fixed point $\omega \in X$.

Proof. Take $\lambda$ such that $0<\lambda<1$. Since $\lim _{n \rightarrow 0} \lambda_{n}=0$, there exists $n_{0} \in \mathbb{N}$ such that $\lambda_{n}<\lambda$ for each $n \geq n_{0}$. Then $q\left(T^{n} x, T^{n} y\right) \preceq$ $\lambda_{n} q(x, y)$ for all $x, y \in X$ whenever $n \geq n_{0}$. In other words, for any $m \geq n_{0}, g=T^{m}$ satisfies

$$
q(g x, g y) \preceq k q(x, y) \text { for all } x, y \in X .
$$

Theorem 2 implies that $g$ has a unique fixed point, say $\omega$. Then $T^{m} \omega=\omega$, implying that $T^{m+1} \omega=T\left(T^{m} \omega\right)=T^{m}(T \omega)=T \omega$ and $T \omega$ is also a fixed point of $g=T^{m}$. Since the fixed point is unique, it follows that $T \omega=\omega$ and $\omega$ is the unique fixed point of $T$.

We now state below a generalization of this theorem.

Theorem 4. Let $(X, q)$ be a bicomplete quasi-cone metric space, $P$ a $K$-normal cone. Suppose that a mapping $T: X \rightarrow X$ is such that for every $n \in \mathbb{N}, T^{n}$ is Lipschitzian and that $\sum_{n=0}^{\infty} \operatorname{Lip}\left(T^{n}\right)<\infty$. Then $T$ has a unique fixed point $x^{*} \in X$. 
Proof. Since for any $n \in \mathbb{N}, T^{n}$ is Lipschitzian, hence there exists $k_{n}:=\operatorname{Lip}\left(T^{n}\right) \geq 0$ such that

$$
q\left(T^{n} x, T^{n} y\right) \preceq k_{n} q(x, y) \text { for all } x, y \in X .
$$

Now let $x \in X$. For any $n, h \in \mathbb{N}$, we have

$$
q\left(T^{n} x, T^{n+h} x\right) \preceq k_{n} q\left(x, T^{h} x\right) \preceq k_{n}\left[\sum_{i=0}^{h-1} q\left(T^{i} x, T^{i+1} x\right)\right] .
$$

Hence

$$
q\left(T^{n} x, T^{n+h} x\right) \preceq k_{n}\left(\sum_{i=0}^{h-1} k_{i}\right) q(x, T x)
$$

since

$$
q\left(T^{i}, T^{i+1} x\right) \preceq k_{i} q(x, T x), \text { for all } i \in \mathbb{N} \text {. }
$$

Since $\sum_{n=0}^{\infty} \operatorname{Lip}\left(T^{n}\right)$ is convergent, then $\lim _{n \rightarrow 0} \operatorname{Lip}\left(T^{n}\right)=0$ and therefore inequality (4) entails that

$$
\left\|q\left(T^{n} x, T^{n+h} x\right)\right\| \leq K k_{n}\left(\sum_{i=0}^{h-1} k_{i}\right)\|q(x, T x)\| \longrightarrow 0 \text { as } n \longrightarrow \infty .
$$

Similarly, one shows that

$$
\left\|q\left(T^{n+h} x, T^{n} x\right)\right\| \leq K k_{n}\left(\sum_{i=0}^{h-1} k_{i}\right)\|q(T x, x)\| \longrightarrow 0 \text { as } n \longrightarrow \infty .
$$

From relations (5) and (6), we conclude that $\left(T^{n} x\right)$ is a bi-Cauchy sequence. Since $(X, q)$ is bicomplete, there exists $x^{*} \in X$ such that $\left(T^{n} x\right)$ converges to $x^{*}$. First let us show that $x^{*}$ is a fixed point of $T$.

On one side, we have

$$
\begin{aligned}
q\left(T^{n-1} x, x^{*}\right) & \preceq q\left(T^{n-1} x, T^{n} x\right)+q\left(T^{n} x, x^{*}\right) \\
& \preceq k_{n-1} q(x, T x)+q\left(T^{n} x, x^{*}\right),
\end{aligned}
$$

and on the other side

$$
\begin{aligned}
q\left(x^{*}, T^{n-1} x\right) & \preceq q\left(x^{*}, T^{n} x\right)+q\left(T^{n} x, T^{n-1} x\right) \\
& \preceq k_{n-1} q(T x, x)+q\left(x^{*}, T^{n} x\right),
\end{aligned}
$$


From (7), we have that

$$
\begin{aligned}
q\left(T x^{*}, x^{*}\right) & \preceq q\left(T x^{*}, T^{n} x\right)+q\left(T^{n} x, x^{*}\right) \\
& \preceq k_{1} q\left(x^{*}, T^{n-1} x\right)+q\left(T^{n} x, x^{*}\right) \rightarrow \theta \text { as } n \rightarrow \infty,
\end{aligned}
$$

i.e

$$
q\left(T x^{*}, x^{*}\right)=\theta .
$$

In the same manner, from (8), we have that

$$
q\left(x^{*}, T x^{*}\right)=\theta .
$$

Hence

$$
q\left(T x^{*}, x^{*}\right)=\theta=q\left(x^{*}, T x^{*}\right) .
$$

This implies, using property $(q 2)$ that $T x^{*}=x^{*}$. So $x^{*}$ is a fixed point of $T$. Moreover, if $z^{*}$ is a fixed point of $T$, then for all $n \geq 1$, we have

$$
q\left(x^{*}, z^{*}\right)=q\left(T^{n} x^{*}, T^{n} z^{*}\right) \preceq k_{n} q\left(x^{*}, z^{*}\right),
$$

and

$$
q\left(z^{*}, x^{*}\right)=q\left(T^{n} z^{*}, T^{n} x^{*}\right) \preceq k_{n} q\left(z^{*}, x^{*}\right) .
$$

Since $\lim _{n \rightarrow 0} \operatorname{Lip}\left(T^{n}\right)=0$, hence $\left\|q\left(x^{*}, z^{*}\right)\right\|=0=\left\|q\left(z^{*}, x^{*}\right)\right\|$ and $x^{*}=z^{*}$. Therefore the fixed point is unique.

In the next section, we give some topological properties of quasi-pseudometric type spaces. Most of them deal with sequences and follow closely the classical properties of sequences pseudometric spaces.

\section{Topology on Quasi-pseudometric type spaces and fixed point results}

3.1. Some topological properties. Let $(X, D, \alpha)$ be a quasi-pseudometric type space. Then for each $x \in X$ and $\epsilon>0$, the set

$$
B_{D}(x, \epsilon)=\{y \in X: D(x, y)<\epsilon\}
$$

denotes the open $\epsilon$-ball at $x$ with respect to $D$. It should be noted that the collection

$$
\left\{B_{D}(x, \epsilon): x \in X, \epsilon>0\right\}
$$

yields a base for the topology $\tau(D)$ induced by $D$ on $X$. In a similar manner, for each $x \in X$ and $\epsilon \geq 0$, we define

$$
C_{D}(x, \epsilon)=\{y \in X: D(x, y) \leq \epsilon\}
$$

known as the closed $\epsilon$-ball at $x$ with respect to $D$. 
Also the collection

$$
\left\{D_{d^{-1}}(x, \epsilon): x \in X, \epsilon>0\right\}
$$

yields a base for the topology $\tau\left(D^{-1}\right)$ induced by $D^{-1}$ on $X$. The set $C_{D}(x, \epsilon)$ is $\tau\left(D^{-1}\right)$-closed, but not $\tau(D)$-closed in general.

The balls with respect to $D$ are often called forward balls and the topology $\tau(D)$ is called forward topology, while the balls with respect to $D^{-1}$ are often called backward balls and the topology $\tau\left(D^{-1}\right)$ is called backward topology.

The topology $\tau(D)$ of a quasi-pseudometric type space $(X, D, \alpha)$ can be defined starting with starting from the family $\Pi_{D}(x)$ of neighbourhoods of an arbitrary point $x \in X$.

$$
\begin{aligned}
V \in \Pi_{D}(x) & \Longleftrightarrow \exists \epsilon>0 \text { such that } B_{D}(x, \epsilon) \subset V \\
& \Longleftrightarrow \exists \epsilon^{\prime}>0 \text { such that } C_{D}(x, \epsilon) \subset V .
\end{aligned}
$$

To see the equivalence in the above definition, we can take for instance $\epsilon^{\prime}=\epsilon / 3$.

The following proposition contains some simple properties of convergent sequences.

Proposition 2. Let $\left(x_{n}\right)$ be a sequence in quasi-pseudometric type space $(X, D, \alpha)$.

(a) If $\left(x_{n}\right)$ is D-convergent to $x$ and $D^{-1}$-convergent to $y$, then $D(x, y)=0$.

(b) If $\left(x_{n}\right)$ is D-convergent to $x$ and $D(y, x)=0$, then $\left(x_{n}\right)$ is also

$D$-convergent to $y$.

\section{Proof.}

(a) Letting $n \rightarrow \infty$ in the inequality

$$
D(x, y) \leq \alpha\left[D\left(x, x_{n}\right)+D\left(x_{n}, y\right)\right]
$$

one obtains $D(x, y)=0$.

(b) The result follows from the relations

$$
D\left(x_{n}, y\right) \leq \alpha\left[D(y, x)+D\left(x, x_{n}\right)\right]=\alpha D\left(x, x_{n}\right) \rightarrow 0 .
$$

Also, the following simple remarks concerning sequences in quasi-pseudometric type spaces are true.

Proposition 3. Let $\left(x_{n}\right)$ be as sequence in a quasi-pseudometric type space $(X, D, \alpha)$.

(a) If $\left(x_{n}\right)$ is left $K$-Cauchy and has a subsequence which is $\tau(D)$-convergent to $x$, then $\left(x_{n}\right)$ is $\tau(D)$-convergent to $x$. 
(b) If $\left(x_{n}\right)$ is left $K$-Cauchy and has a subsequence which is $\tau\left(D^{-1}\right)$-convergent to $x$, then $\left(x_{n}\right)$ is $\tau\left(D^{-1}\right)$-convergent to $x$.

Proof. (a) Suppose that $\left(x_{n}\right)$ is left $K$-Cauchy and $\left(x_{n_{k}}\right)$ is a subsequence of $\left(x_{n}\right)$ such that $\lim _{k \rightarrow \infty} D\left(x, x_{n_{k}}\right)=0$. For $\epsilon>0$ choose $n_{0}$ such that $n_{0} \leq$ $m \leq n$ implies $D\left(x_{m}, x_{n}\right)<\epsilon / \alpha$, and let $k_{0} \in \mathbb{N}$ be such that $n_{k_{0}} \geq n_{0}$ and $D\left(x, x_{n_{k}}\right)<\epsilon / \alpha$ for all $k \geq k_{0}$. Then, for $n \geq n_{k_{0}}, D\left(x, x_{n}\right) \leq \alpha\left[D\left(x, x_{n_{k_{0}}}+\right.\right.$ $\left.D\left(x_{n_{k_{0}}}, x_{n}\right)\right]<2 \epsilon$.

(b) Reasoning similarly, for $n \geq n_{k_{0}}$ let $k \in \mathbb{N}$ such that $n_{k} \geq n$. Then $D\left(x_{n}, x\right) \leq \alpha\left[D\left(x_{n}, x_{n_{k}}\right)+D\left(x_{n_{k}}, x\right)\right]<2 \epsilon$.

The proof $\mathrm{f}$ the following proposition is trivial and shall then be omitted.

Proposition 4. If a sequence $\left(x_{n}\right)$ in a quasi-pseudometric type space $(X, D, \alpha)$, satisfies

$$
\sum_{n=0}^{\infty} D\left(x_{n}, x_{n+1}\right)<\infty,
$$

then $\left(x_{n}\right)$ is left K-Cauchy.

Definition 15. A subset $Y$ of a quasi-pseudometric type space $(X, D, \alpha)$ is called precompact if for every $\epsilon>0$ there exists a finite subset $Z$ of $Y$ such that

$$
Y \subset \cup\left\{B_{D}(z, \epsilon): z \in Z\right\}
$$

If for every $\epsilon>0$ there exists a finite subset $Z$ of $X$ such that (9) holds, then the set $Y$ is called outside precompact. One obtains the same notions if one works with closed balls $C_{D}(z, \epsilon) z \in Z$.

Obviously a precompact set is outside precompact, but the converse is not true. We then have the following characterization.

Proposition 5. Let $(X, D, \alpha)$ be a quasi-pseudometric type space. A subset $Y$ of $X$ is precompact if and only if for every $\epsilon>0$ there is a finite subset $\left\{x_{1}, x_{2}, \cdots, x_{n}\right\} \subset X$ such that $Y \subset \cup_{i=1}^{n} B_{D}\left(x_{i}, \epsilon\right)$ and $Y \cap B_{D^{-1}}\left(x_{i}, \epsilon\right) \neq \emptyset$ for all $i=1,2, \cdots, n$.

Proof. For $\epsilon>0$, let $\left\{x_{1}, x_{2}, \cdots, x_{n}\right\} \subset X$ such that the conditions hold for $\epsilon / 2 \alpha$. If $y_{i} \in Y \cap B_{D^{-1}}\left(x_{i}, \epsilon / 2 \alpha\right), i=1,2, \cdots, n$, then $Y \subset \cup_{i=1}^{n} B_{D}\left(x_{i}, \epsilon\right)$.

Indeed, for any $y \in Y$ there exists $k \in\{1,2, \cdots, n\}$ such that $D\left(x_{k}, y\right)<$ $\epsilon / 2 \alpha$, implying

$$
D\left(y_{k}, y\right) \leq \alpha\left[D\left(y_{k}, x_{k}\right)+D\left(x_{k}, y\right)\right]=\alpha\left[D^{-1}\left(x_{k}, y_{k}\right)+D\left(x_{k}, y\right)\right]<\epsilon .
$$


3.2. Fixed point results. We start with the following lemma and repeat the proof as it is in [4].

Lemma 2 (Compare [4] Lemma 38). Let $\left(y_{n}\right)$ be a sequence in a quasipseudometric type space $(X, D, \alpha)$ such that

$$
D\left(y_{n}, y_{n+1}\right) \leq \lambda D\left(y_{n-1}, y_{n}\right)
$$

for some $\lambda>0$ with $\lambda<\min \{1,1 / \alpha\}$. Then $\left(y_{n}\right)$ is left $K$-Cauchy.

Proof. Let $m<n \in \mathbb{N}$. From the condition (D2) in the definition of a quasi-pseudometric type, we can write:

$$
\begin{aligned}
D\left(y_{m}, y_{n}\right) \leq & \alpha\left[D\left(y_{m}, y_{m+1}\right)+D\left(y_{m+1}, y_{n}\right)\right] \\
\leq & \alpha D\left(y_{m}, y_{m+1}\right)+\alpha^{2} D\left(y_{m+1}, y_{m+2}\right)+\alpha^{2} D\left(y_{m+2}, y_{n}\right) \\
& \vdots \\
\leq & \alpha D\left(y_{m}, y_{m+1}\right)+\alpha^{2} D\left(y_{m+1}, y_{m+2}\right)+\cdots \\
& +\alpha^{n-m-1} D\left(y_{n-2}, y_{n-1}\right)+\alpha^{n-m} D\left(y_{n-1}, y_{n}\right) .
\end{aligned}
$$

From (10) and $\lambda<\frac{1}{\alpha}$, the above becomes

$$
\begin{aligned}
D\left(y_{m}, y_{n}\right) & \leq\left(\alpha \lambda^{m}+\alpha^{2} \lambda^{m+1}+\cdots+\alpha^{n-m} \lambda^{n-1}\right) D\left(y_{0}, y_{1}\right) \\
& \leq \alpha \lambda^{m}\left(1+\alpha \lambda+\cdots+(\alpha \lambda)^{n-1-m}\right) D\left(y_{0}, y_{1}\right) \\
& \leq \frac{\alpha \lambda^{m}}{1-\alpha \lambda} D\left(y_{0}, y_{1}\right) \longrightarrow 0 \text { as } m \longrightarrow \infty .
\end{aligned}
$$

It follows that $\left(y_{n}\right)$ is left $K$-Cauchy. Similarly,

Lemma 3. Let $\left(y_{n}\right)$ be a sequence in a quasi-pseudometric type space $(X, D, \alpha)$ such that

$$
D^{-1}\left(y_{n}, y_{n+1}\right) \leq \lambda D^{-1}\left(y_{n-1}, y_{n}\right)
$$

for some $\lambda>0$ with $\lambda<\min \{1,1 / \alpha\}$. Then $\left(y_{n}\right)$ is right $K$-Cauchy.

We now state our first fixed point result.

Theorem 5. Let $(X, D, \alpha)$ be a $T_{0}$-quasi-pseudometric type space. Suppose that $f, g: X \rightarrow X$ are mappings such that

$$
D(f x, f y) \leq k D(g x, g y) \text { for all } x, y \in X,
$$

where $k<\min \{1,1 / \alpha\}$. If the range of $g$ contains the range of $f$ and $g(X)$ is bicomplete, then $f$ and $g$ have a unique point of coincidence. Moreover if $f$ and $g$ are weakly compatible, then $f$ and $g$ have a unique common fixed point. 
Proof. Take an arbitrary $x_{0} \in X$. Choose a point $x_{1}$ in $X$ such that $f\left(x_{0}\right)=g\left(x_{1}\right)$. This can be done, since $f(X) \subset g(X)$. Iterating this process, once $x_{n}$ is chosen in $X$, we can obtain $x_{n+1}$ in $X$ such that $f\left(x_{n}\right)=g\left(x_{n+1}\right)$. Then

$$
\begin{aligned}
D\left(g x_{n}, g x_{n+1}\right) & =D\left(f x_{n-1}, f x_{n}\right) \leq k D\left(g x_{n-1}, g x_{n}\right) \\
& \leq k^{2} D\left(g x_{n-2}, g x_{n-1}\right) \leq \ldots \leq k^{n} D\left(g x_{0}, g x_{1}\right) .
\end{aligned}
$$

i.e.

$$
D\left(g x_{n}, g x_{n+1}\right) \leq k^{n} D\left(g x_{0}, g x_{1}\right) .
$$

Similarly,

$$
D\left(g x_{n+1}, g x_{n}\right) \leq k^{n} D\left(g x_{1}, g x_{0}\right) .
$$

Hence $\left(g x_{n}\right)$ is a bi-Cauchy sequence. Since $g(X)$ is bicomplete, there exists $x^{*} \in g(X)$ such that $\left(g x_{n}\right) D^{s}$-converges to $x^{*}$. In other words, there is a $p^{*} \in X$ such that $\left(g x_{n}\right)$ converges to $g\left(p^{*}\right)=x^{*}$.

Moreover

$$
D\left(g x_{n}, f p^{*}\right)=D\left(f x_{n-1}, f p^{*}\right) \leq k D\left(g x_{n-1}, g p^{*}\right) \longrightarrow 0, \text { as } n \longrightarrow,
$$

In the same way, we establish that $D\left(f p^{*}, g x_{n}\right) \longrightarrow 0$ as $n \longrightarrow \infty$, to then conclude that $g x_{n} \longrightarrow f p^{*}$. The uniqueness of the limit implies that $f p^{*}=g p^{*}$. We finish the proof by showing that $f$ and $g$ have a unique point of coincidence. For this, assume $z^{*} \in X$ is a point such that $f z^{*}=g z^{*}$.

Now

$$
D\left(g z^{*}, g p^{*}\right)=D\left(f z^{*}, f p^{*}\right) \leq k D\left(g z^{*}, g p^{*}\right),
$$

which gives $D\left(g z^{*}, g p^{*}\right)=0$. On the other hand, by the same reasoning, it also clear that $D\left(g p^{*}, g z^{*}\right)=0$. By property the $T_{0}$-condition, $g z^{*}=g p^{*}$. From Proposition $1, f$ and $g$ have a unique common fixed point.

Theorem 6. Let $(X, D, \alpha)$ be a $T_{0}$-quasi-pseudometric type space. Suppose that $f, g: X \rightarrow X$ are mappings such that Suppose that mappings $f, g: X \rightarrow X$ satisfy the contractive condition

$$
D(f x, f y) \leq k[D(f x, g y)+D(g x, f y)] \text { for all } x, y \in X,
$$

where $k \geq 0$ such that $\frac{k}{1-k}<\min \{1,1 / \alpha\}$. If the range of $g$ contains the range of $f$ and $g(X)$ is bicomplete, then $f$ and $g$ have a unique coincidence point in $X$. Moreover if $f$ and $g$ are weakly compatible, then $f$ and $g$ have a unique common fixed point. 
Take an arbitrary $x_{0} \in X$. Choose a point $x_{1}$ in $X$ such that $f\left(x_{0}\right)=$ $g\left(x_{1}\right)$. This can be done, since $f(X) \subset g(X)$. Iterating this process, once $x_{n}$ is chosen in $X$, we can obtain $x_{n+1}$ in $X$ such that $f\left(x_{n}\right)=g\left(x_{n+1}\right)$. Then

$$
\begin{aligned}
D\left(g x_{n}, g x_{n+1}\right) & =D\left(f x_{n-1}, f x_{n}\right) \leq k\left[D\left(f x_{n-1}, g x_{n}\right)+D\left(g x_{n-1}, f x_{n}\right)\right] \\
& \leq k D\left(g x_{n-1}, g x_{n+1}\right) \\
& \leq k\left[D\left(g x_{n-1}, g x_{n}\right)+D\left(g x_{n}, g x_{n+1}\right)\right]
\end{aligned}
$$

which entails that

$$
D\left(g x_{n}, g x_{n+1}\right) \leq \frac{k}{1-k}\left(g x_{n-1}, g x_{n}\right) .
$$

Similarly,

$$
D\left(g x_{n+1}, g x_{n}\right) \leq \frac{k}{1-k} D\left(g x_{n}, g x_{n-1}\right) .
$$

Hence $\left(g x_{n}\right)$ is a bi-Cauchy sequence. Since $g(X)$ is bicomplete, there exists $x^{*} \in g(X)$ such that $\left(g x_{n}\right) D^{s}$-converges to $x^{*}$. In other words, there is a $p^{*} \in X$ such that $\left(g x_{n}\right)$ converges to $g\left(p^{*}\right)=x^{*}$.

Moreover since

$$
D\left(g x_{n}, f p^{*}\right)=D\left(f x_{n-1}, f p^{*}\right) \leq k\left[D\left(f x_{n-1}, g p^{*}\right)+D\left(g x_{n-1}, f p^{*}\right)\right],
$$

we get that

$$
D\left(g p^{*}, f p^{*}\right) \leq k D\left(g p^{*}, f p^{*}\right)
$$

which implies that $D\left(g p^{*}, f p^{*}\right)=0$.

In the same way, we establish that $D\left(f p^{*}, g p^{*}\right)=0$, to then conclude that $f p^{*}=g p^{*}$.

We finish the proof by showing that $f$ and $g$ have a unique point of coincidence. For this, assume $z^{*} \in X$ is a point such that $f z^{*}=g z^{*}$. Now

$$
\begin{aligned}
D\left(g z^{*}, g p^{*}\right)=D\left(f z^{*}, f p^{*}\right) & \leq k\left[D\left(f z^{*}, g p^{*}\right)+D\left(g z^{*}, f p^{*}\right)\right] \\
& \leq 2 k D\left(g z^{*}, g p^{*}\right),
\end{aligned}
$$

which gives $D\left(g z^{*}, g p^{*}\right)=0$. On the other hand, by the same reasoning, it also clear that $D\left(g p^{*}, g z^{*}\right)=0$. Therefore $g z^{*}=g p^{*}$. From Proposition 1 , $f$ and $g$ have a unique common fixed point.

Theorem 7. Let $(X, D, \alpha)$ be a $T_{0}$-quasi-pseudometric type space. Suppose that $f, g: X \rightarrow X$ are mappings such that

$$
D(f x, f y) \leq \lambda D(g x, g y)+\gamma D(f x, g y) \text { for all } x, y \in X .
$$

where $\lambda, \gamma$ are positive constants such that $\lambda+2 \gamma<\min \{1,1 / \alpha\}$. If the range of $g$ contains the range of $f$ and $g(X)$ is bicomplete, then $f$ and $g$ have $a$ unique coincidence point in $X$. Moreover if $f$ and $g$ are weakly compatible, then $f$ and $g$ have a unique common fixed point. 
Proof. Take an arbitrary $x_{0} \in X$. Choose a point $x_{1}$ in $X$ such that $f\left(x_{0}\right)=g\left(x_{1}\right)$. This can be done, since $f(X) \subset g(X)$. Iterating this process, once $x_{n}$ is chosen in $X$, we can obtain $x_{n+1}$ in $X$ such that $f\left(x_{n}\right)=g\left(x_{n+1}\right)$. Then

$$
\begin{aligned}
D\left(g x_{n}, g x_{n+1}\right)=D\left(f x_{n-1}, f x_{n}\right) & \leq \lambda D\left(g x_{n-1}, g x_{n}\right)+\gamma D\left(f x_{n-1}, g x_{n}\right) \\
& \leq \lambda D\left(g x_{n-1}, g x_{n}\right) .
\end{aligned}
$$

Therefore $\left(g x_{n}\right)$ is a left $K$-Cauchy sequence. In a similar manner, we establish that $\left(g x_{n}\right)$ is also a right $K$-Cauchy sequence. Hence $\left(g x_{n}\right)$ is a bi-Cauchy sequence. Since $g(X)$ is bicomplete, there exists $x^{*} \in g(X)$ such that $\left(g x_{n}\right) D^{s}$-converges to $x^{*}$. In other words, there is a $p^{*} \in X$ such that $\left(g x_{n}\right)$ converges to $g\left(p^{*}\right)=x^{*}$.

Moreover since

$$
D\left(g x_{n}, f p^{*}\right)=D\left(f x_{n-1}, f p^{*}\right) \leq \lambda D\left(g x_{n-1}, g p^{*}\right)+\gamma D\left(f x_{n-1}, g p^{*}\right)
$$

we get that $D\left(g p^{*}, f p^{*}\right)=0$. On the other hand, by the same reasoning, it is also clear that $D\left(f p^{*}, g p^{*}\right)=0$. Hence $f p^{*}=g p^{*}$.

We finish the proof by showing that $f$ and $g$ have a unique point of coincidence. For this, assume $z^{*} \in X$ is a point such that $f z^{*}=g z^{*}$. Now

$$
\begin{aligned}
D\left(g z^{*}, g p^{*}\right)=D\left(f z^{*}, f p^{*}\right) & \leq \lambda D\left(g z^{*}, g p^{*}\right)+\gamma D\left(f z^{*}, g p^{*}\right) \\
& \leq(\lambda+\gamma) D\left(g z^{*}, g p^{*}\right),
\end{aligned}
$$

which gives $D\left(g z^{*}, g p^{*}\right)=0$. On the other hand, by the same reasoning, it also clear that $D\left(g p^{*}, g z^{*}\right)=0$. Hence $g z^{*}=g p^{*}$. From Proposition 1, $f$ and $g$ have a unique common fixed point.

We now give an example to illustrate Theorems 5,7 .

Example 2. Let $X=\mathbb{R}, D(x, y)=\max \{x-y, 0\}$ whenever $x, y \in \mathbb{R}$, $f(x)=2 x^{2}+4 x+1$ and $g(x)=3 x^{2}+6 x+2$. Then it easy to see that

$$
f(X)=g(X)=[1, \infty) \text { is bicomplete. }
$$

All the conditions of Theorems 5, 7 are satisfied. Indeed:

- for Theorem 5 , take $k \in\left[\frac{2}{3}, 1\right)$

- for Theorem 7 , take $\lambda \in\left[\frac{2}{3}, 1\right), \gamma=0$.

$f$ and $g$ become weakly compatible and we obtain a unique point of coincidence and a unique common fixed point $-1=f(-1)=g(-1)$. 
Corollary 1. Let $(X, D, \alpha)$ be a $T_{0}$-quasi-pseudometric type space. Suppose that mappings $f, g: X \rightarrow X$ satisfy the contractive condition

$$
D(f x, f y) \leq \alpha[D(g x, g y)+D(f x, g y)] \text { for all } x, y \in X .
$$

where $0<\alpha<\min \{1,1 / 3 \alpha\}$. If the range of $g$ contains the range of $f$ and $g(X)$ is bicomplete, then $f$ and $g$ have a unique coincidence point in $X$. Moreover if $f$ and $g$ are weakly compatible, then $f$ and $g$ have a unique common fixed point.

Theorem 8. Let $(X, D, \alpha)$ be a $T_{0}$-quasi-pseudometric type space. Suppose that $f, g: X \rightarrow X$ are mappings such that

$$
D(f x, f y) \leq \lambda D(g x, g y)+\gamma D(g x, f y) \text { for all } x, y \in X .
$$

where $\lambda, \gamma$ are positive constants such that $\lambda+2 \gamma<\min \{1,1 / \alpha\}$. If the range of $g$ contains the range of $f$ and $g(X)$ is bicomplete, then $f$ and $g$ have $a$ unique coincidence point in $X$. Moreover if $f$ and $g$ are weakly compatible, then $f$ and $g$ have a unique common fixed point.

Corollary 2. Let $(X, D, \alpha)$ be a $T_{0}$-quasi-pseudometric type space. Suppose that mappings $f, g: X \rightarrow X$ satisfy the contractive condition

$$
D(f x, f y) \leq \lambda[D(g x, g y)+D(g x, f y)] \text { for all } x, y \in X .
$$

where $0<\lambda<\min \{1,1 / 3 \alpha\}$. If the range of $g$ contains the range of $f$ and $g(X)$ is bicomplete, then $f$ and $g$ have a unique coincidence point in $X$. Moreover if $f$ and $g$ are weakly compatible, then $f$ and $g$ have a unique common fixed point.

\section{References}

[1] Abbas M., Jungck G., Common fixed point results for noncommuting mappings without continuity in come metric spaces, J. Math. Anal. App., 341(2008), 416-420.

[2] Gaba Y.U., Startpoints and $(\alpha, \gamma)$-contractions in quasi-pseudometric spaces, Journal of Mathematics, Vol. 2014 (2014), Article ID 709253, 8 pages. http://dx.doi.org/10.1155/2014/709253.

[3] Huang L.-G., Zhang X., Cone metric spaces and fixed point theorems of contractive mappings, Journal of Mathematical Analysis and Applications, $332(2)(2007), 1468-1476$.

[4] Kazeem E.F., Agyingi C.A., Gaba Y.U., On quasi-pseudometric type spaces, Chinese Journal of Mathematics, 2014, Article ID 198685, 7 pages, 2014. doi:10.1155/2014/198685. 
[5] Khamsi M.A., Remarks on cone metric spaces and fixed point theorems of contractive mappings, Fixed Point Theorem and Application, 2010(2010), 7 pages, doi:10.1155/2010/315398.

[6] Wilson W.A., On semi-metric spaces, American Journal of Mathematics, 53(2)(1931), 361-373.

[7] WŁodarczyk K., Plebaniak R., Asymmetric structures, discontinuous contractions and iterative approximation of fixed and periodic points, Fixed Point Theory and Applications, 2013 (2013) 128, 1-18. http://dx.doi.org /10.1186/1687-1812-2013-128.

[8] Wøodarczyk K., Plebaniak R., New completeness and periodic points of discontinuous contractions of Banach type in quasi-gauge spaces without Hausdorff property, Fixed Point Theory and Applications, 2013 (2013) 289, 1-27. http://dx.doi.org/10.1186/1687-1812-2013-289.

\author{
Yá́ UlRich Gaba \\ Department of Mathematics and Applied Mathematics, \\ UNIVERSITY OF CAPE TOWN \\ Rondebosch 7701, South AfricA \\ e-mail: gabayae2@gmail.com
}

Received on 14.08.2015 and, in revised form, on 13.04.2016. 